\title{
MJMR MANAGEMENT OF LARGE SIZE AND MULTIPLE BENIGN BREAST LESIONS USING ONCOPLASTIC SURGICAL SKILLS
}

\author{
Saira Saleem ${ }^{1 *}$, Farhan Javed ${ }^{2}$, Irshad Ahmad ${ }^{3}$. \\ ${ }^{1}$ Associate Professor Surgery, Madina Teaching Hospital \& University Medical and Dental College, \\ Faisalabad, Pakistan \\ ${ }^{2}$ Assistant Professor Surgery, Madina Teaching Hospital \& University Medical and Dental College, \\ Faisalabad, Pakistan. \\ ${ }^{3}$ Head of department and Professor of Surgery, Madina Teaching Hospital \& University Medical and \\ Dental College Faisalabad, Pakistan. \\ Corresponding Author E-mail: dsairaijaz32@yahoo.com
}

\begin{abstract}
Objective: Management of multiple and large size benign breast lesions is challenging. Usual excision techniques are associated with unacceptable breast deformity in such cases. However, these can effectively be managed with minimum breast deformity using oncoplastic skills used for breast cancer. Our objective was to evaluate the oncological outcome and patient satisfaction of managing large and multiple benign breast lesions using oncoplastic surgical skills. Methods: This was a descriptive, prospective study conducted in department of Surgery, Madina Teaching Hospital, Faisalabad, Pakistan from January 2018 to December 2019. Females with age range of 12 to 60 years were included in the study. Different oncoplastic techniques were used depending on location and size of tumour as well as size and degree of breast ptosis.Main outcome measures were tumour recurrence and patient satisfaction with the cosmetic outcome. Results: Twenty-eight female patients with large size $(>5 \mathrm{~cm})$ or multiple benign breast tumors were managed. Most common techniques used were round block mammoplasty and wise pattern reduction mammoplasty. Giant fibroadenoma (35.7\%), benign phyllodes tumour (14\%) and ductal hyperplasia (usual 10.7\% \& atypical 7\%) were the most common histological findings. No tumour recurrence was seen during our study period and minimum scarring and no breast deformity in these cases, gained high patient satisfaction. Conclusion: Oncoplastic skills achievefavourable aesthetic and oncological results for large and multiple benign breast tumours.
\end{abstract}

\section{Keywords: Giant Fibroadenoma, PhyllodesTumour, Oncoplastic Skills, Patient Satisfaction}

\section{INTRODUCTION}

Vast majority of the diseases seen in the breast are benign. Benign breast disorders cover a wide range of histologic entities that are usually sub divided into nonproliferative lesions, proliferative breast lesions without atypia, and proliferative breast lesions with atypia (Okoth et al., 2013). Common benign lesions such as fibroadenoma or mammary adenosis usually present as multiple and bilateral masses (Zhang et al., 2019; Neal et al., 2014). Incidence of benign lesions like fibroadenoma increase during the second decade of life. Benign proliferative breast lesions are usually observed in women of 30 to 40 years age group. Large size of these lesions sometime cause significant breast asymmetry (Chirappapha et al., 2016). Another category of breast tumours is the phyllodes tumour that is a fibroepithelial-tumour with a spectrum of changes. It is usually difficult to differentiate a benign phyllodes tumor from a fibroadenoma. Differentiation of phyllodes tumour is important as it should be excised completely with a clear margin to obviate any chance of local recurrence (Guray et al., 2006).

Benign tumours are usually treated by surgical excision. Simple excision may be satisfactory for patients with small to moderate-sized lesions; however, excision of large and multiple tumours may be challenging as 
simple excision leads to a displeasing, loose, ptotic breast (Simmons et al., 2000). Oncoplastic surgery has become increasingly popular in recent years combining the principles of oncology and plastic surgery. Oncoplastic breast surgery can be defined as a tumorfocused immediate breast reconstruction allowing for wider volume excision with no aesthetic compromise (Najafi et al., 2019).

In large or multiple benign lesions, like giant fibro adenoma the surgeon is faced with a reconstructive challenge similar to when a large malignant breast neoplasm is removed (Hiller et al., 2018). Oncoplastic breast surgery is an important revolution in breast surgery in which we can perform a wide tumour resection even for large size and poorly located cancers (Saleem et al., 2019). Some of the patients have associated macro mastia. Combining reduction mammoplasty with mastopexy relieve the patients from their symptoms related to macro mastia such as back and neck pain, which improves their overall level of satisfaction (Yazar et al., 2018).

In this study we used onco-plastic skills for excision of a variety of large sized and multiple benign breast tumours to maintain aesthetically natural breast form.

\section{METHODOLOGY}

This descriptive, prospective study was conducted between January 2018 to December 2019. 28 patients with benign breast tumours that were of large size or multiple in number were included in the study. Some of these cases had associated breast hypertrophy. Benign nature of the lesion was assessed by clinical examination and further evaluated by radiological and cytological investigations. In some cases, like phyllodes tumour or atypical hyperplasia tru-cut biopsy was also done for confirmation and histological diagnosis.

Patient was explained about operative procedure and possible complications and informed consent was taken. Approval by ethical review committee for research was taken before the start of study.

Preoperative markings were done in upright position of the patients. Different oncoplastic surgical techniques were used depending on tumour location, number, breast size and associated hypertrophy. Pedicle for nipple areola complex was selected according to location of tumour, e.g., superior pedicle, inferior pedicle and superomedial pedicles were selected in case of inferior, superior or superolateral tumors respectively. In most of the cases round block technique was used. In some cases, with giant tumour or associated breast hypertrophy wise pattern, reduction mammoplasty technique was used.

In cases with diagnosis of phyllodes tumour, wide excision of tumour taking $1 \mathrm{~cm}$ tumour free margin was done. In patients with multiple tumours at lower inner or outer quadrant, an inframammary or lateral crease incision and a retromammary plane for dissection was used for better cosmetic results.

Following surgery patients were assessed for cosmetic outcome, postoperative complications, recurrence and patient's satisfaction with the outcome. Follow up was done initially weekly in first month and then 6 monthly. Patient satisfaction with the aesthetic outcome was verbally obtained; 0 : Not satisfied, 1: Low, 2: Moderate, 3: Highly satisfied (Yazar et al., 2018).

\section{Statistical Analysis}

Numerical (continuous) variables like age, BMI, hospital stay, surgery time, tumour size, weight of the excised specimen, patient cosmetic score; were presented as Mean $\pm \mathrm{SD}$. For qualitative variables like associated comorbidity (Diabetes mellitus), site of tumour, post op complications, histopathology findings, tumour recurrence; frequency and percentages were calculated and presented in tables and graphs.

Descriptive analysis was done for the above-listed variables. Statistical analysis was performed using Microsoft Excel 2010 (Microsoft Corporation, Redmond, WA, USA).

\section{RESULTS}

Most of the patients were young with a mean age of 32.5 $(\mathrm{SD}=3.5)$ and $\mathrm{BMI}$ of $23.2(\mathrm{SD}=1.4)$. Table 1 shows the descriptive analysis of study results.In majority of the patients, presentation was late. Some of them came earlier and were advised surgery but they neglected it for many years, considering it a benign lesion and tumour became giant size or multiple in number. 
Table 1: Descriptive analysis of the study results

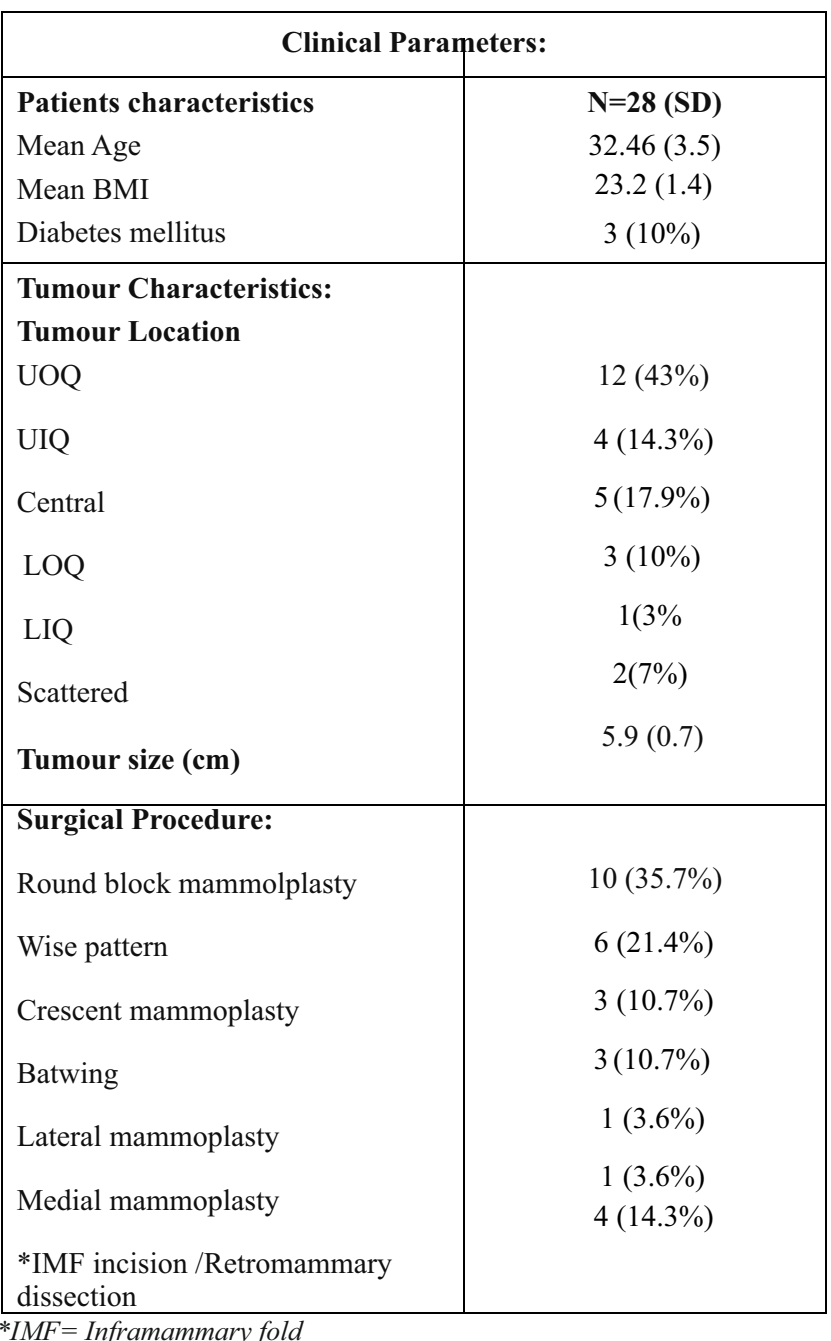

Surgical excision and mammoplasty was done for the patients. Most common techniques used were round block mammoplasty and wise patterns reduction mammoplasty (Fig 1,2).

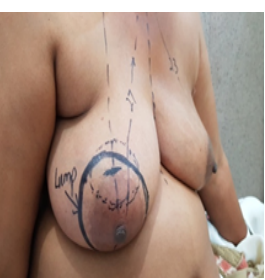

(a)

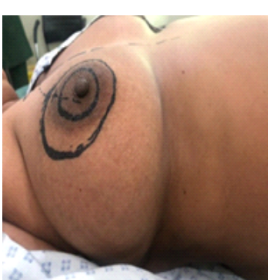

(b)

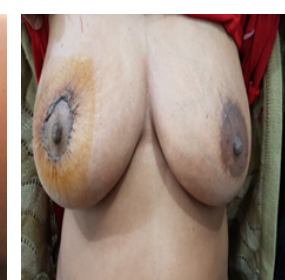

(c)
Figure 1: (a): Pre operative lump and breast assymtry, (b): round bock mammoplasty marking, (c): Post operative showing normal shape and symmetry of breasts

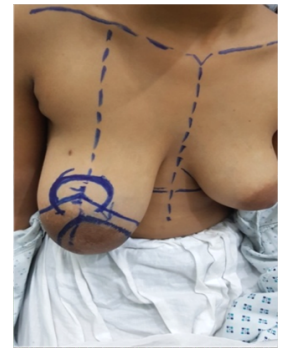

(a)

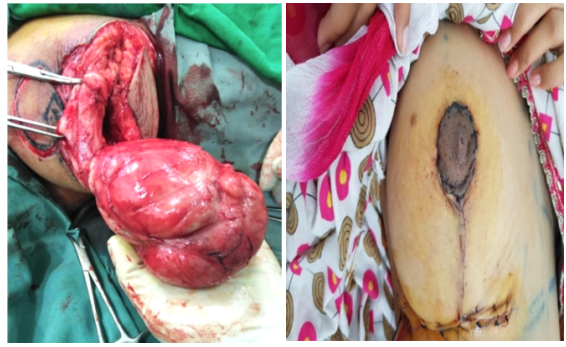

(b)

(c)
Figure 2: A case of Juvenile hypertrophied breast with giant fibroadenoma (a): preoperative marking, (b): Intraoperative lump excision, (c): Postoperative pic

Some of our patients had marked unilateral breast hypertrophy along with giant fibroadenoma. 2 of these cases were juvenile hypertrophy, 2 lactational and one adult hypertrophied breast. This associated macromastia was managed by reduction mammoplasty. (Fig2,3).

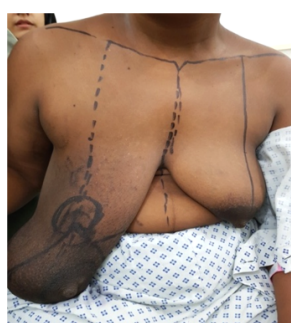

(a)

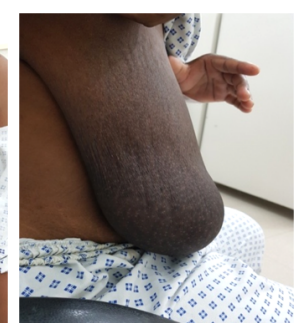

(b)

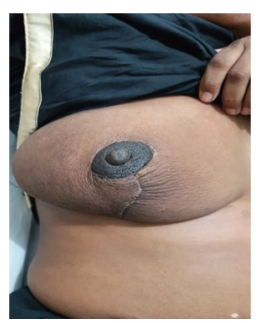

(c)
Fig 3 a-c: A case of giant fibroadenoma with marked lactational hypertrophied breast (a): Preoperative marking, (b): Preoperative image, (c): Post operative image at $7^{\text {th }}$ post op day.

In young girls with multiple fibroadenomas, a retromammary approach was used to deliver fibroadenomas, that was least mutilating and gave very good cosmetic results.

The average operative time was $43.4 \mathrm{~min}$. The average postoperative duration of hospitalization was $2.7 \pm 2.1$ days. Histopathology showed most of the cases of large size lumps to be, giant fibroadenoma followed by phyllodes tumour, ductal hyperplasia, tuberculous mass,ductectasia and periductal mastitis and complex cyst. In all the 5 cases of benign phyllodes tumour, histopathology showed no margin involvement. Regarding post operative complications, minor issues like seroma formation was seen in 4 patients (11.2\%). However, no case of mortality, postoperative bleeding/ 
haematoma, severe breast deformity, or other major complication was observed.

Regarding final outcome at a maximum follow up of 18 months, all our patients were highly satisfied with the cosmetic appearance and no tumour recurrence was seen in any patient

\section{DISCUSSION}

Non-cancerous diseases of the breast have assumed increasing importance because of the public awareness of breast cancer(Okothet al.,2013). Benign tumours of breast associated with significant breast enlargement pose a challenge in surgical management, as usual excision leaves the breast with considerable defect and asymmetry (Pukancsik et al., 2016; Bertozzi et al., 2017). Oncoplastic breast surgery has gained a widespread popularity during the last two decades and it has enabled surgeons to perform breast conservation in large tumors (Kaviani et al., 2015).

Most common oncoplastic techniques used in our study were round block and wise pattern reduction mammoplasty techniques. Nour and colleagues also described similar techniques for the management of multiple and large benign tumours (Nour et al., 2011). Few other techniques are also described in literature like Dolmans et al described nipple sparing subcutaneous mastectomy and implant for the management of a case of giant fibroadenoma (Dolmans et al., 2007). In our study, most of the patients had good volume or hypertrophied breast and desired breast reduction rather than augmentation.

Fibroadenoma was the most common benign tumour variety in our study. Special variants of fibroadenoma described in literature include giant fibroadenoma, juvenile fibroadenoma, fibroadenoma in pregnancy and lactation, and multiple fibroadenoma. Juvenile fibroadenoma is described as a giant fibroadenoma occurring in young or adolescent females (between 11 and 18 years) (Wendy et al., 2011). In our study we encountered and managed all these varieties of fibroadenoma. Five of our cases had lump with marked hypertrophied breast. Three of the patients had juvenile fibroadenoma (age 12 to 14 years) and other two had lactational hypertrophy. These cases had significant ptosis and skin stretch (Fig2,3). All of these cases were managed by a combination of mastopexy and reduction mammoplasty techniques. This combination effectively reduces the number of aesthetically unpleasing results of surgery in patients with macromastia and at the same time maintains adequate tumour free margins (Yazar et al., 2018). King KS also reported a case of giant juvenile fibroadenoma with associated breast hypertrophy that was managed by same principles and technique (King $e t$ al., 2017).

In our study we managed 5 patients with benign phyllodes tumour. All the cases were managed by wide local excision taking $1 \mathrm{~cm}$ gross tumour free margin, as literature suggest (de Rooset al.,1999;Reinfuss et al.,1996). In these cases, if histopathology shows a narrow margin or no margin, re-excision is needed to avoid chances of recurrence (Chirappapha et al., 2016). In all our cases margins were wide and no re-excision was needed and no recurrence was seen at a maximum follow up of $18^{\text {th }}$ months.

Regarding outcome, all of our patients were highly satisfied with the cosmetic appearance. Especially the patients with macromastia were relieved of the symptoms like backache and neck pain that improved their quality of life and overall satisfaction as reported by Yazar SK and colleagues(Yazar et al., 2018).

Oncoplastic surgery techniques used for carcinoma of the breast are very effective and reliable option for treating special therapeutic issues posed by benign breast diseases (Hiller et al., 2018)as seen in this study.

\section{CONCLUSION}

Resection of large and multiple benign tumors is challenging. Oncoplastic surgical techniques are a valuable component of breast surgery in such patients, being oncologically safe and maintain acceptable cosmesis at the same time.

\section{CONFLICT OFINTERESTS}

The authors declare that they have no conflict of interest.

\section{REFERENCES}

Bertozzi, N., Pesce, I.M., Santip, L., Raposio, E.(2017)'Oncoplastic breast surgery: comprehensive review',European Review for Medical and Pharmacological Sciences21(11), pp: 2572-2585 
Chirappapha,P., Lertsithichai, P., Sukarayothin, T., Leesombatpaiboon, M., Supsamutchai, C., KongdonY. (2016) 'Special Therapeutic problems in benign breast conditions', Gland Surg; 5(1), pp: 75-82.

de Roos, W.K., Kaye, P\& Dent DM. (1999)'Factors leading to local recurrence or death after surgical resection of phyllodes tumours of the breast', British Journal of Surgery; 86(3), pp:396-399.

Dolmans, H.C.G. Guido., Hoogbergen, M.H \& van Rappard,J.H.A. (2007)'Giant fibroadenoma of one breast: Immediate bilateral reconstruction', Journal of Plastic, Reconstructive \& Aesthetic Surgery; 60 (10), pp. 1156-1157.

Guray, M and Sahin, A.A. (2006) 'Benign breast diseses : Classification, Diagnosis and management', The Oncologist; 11(5), pp: 435-449

Hiller, A., Lee, T.J., Henderson, J., Ajkay, N., Wilhelmi, B.J. (2018)'Oncoplasticreduction pattern technique following removal of giant fibroadenoma', Eplasty; 18(4), pp: 28-34

Kaviani, A., Najafi, M \& Salmon, J. (2015) 'Oncological Outcome of Oncoplastic Breast Surgery: A Review of the Literature', Archives of Breast Cancer; 2(1), pp: 5-14.

King, K.S., Harrington, M.A., Kassira N. (2017) 'Recurrent giant juvenile fibroadenoma', Journal of Pediatric Surgery Case Reports; 26, pp:42-45

Neal,L., Sandhu, N.P., Hieken, T.J., Glazebrook, K.N., Mac Bride, M.B., Dilaveri,C.A, Wahner-Roedler, D.L., Ghosh, K., Visscher, D.W. (2014) 'Diagnosis and management of benign, atypical and indeterminate breast lesions detected on core needle biopsy', Mayo Clinic Proceedings; 89(4), pp:536-547

Okoth, C., Moses,Galukande, M., Jombwe, J
\&Wamala, D.(2013) 'Benign proliferative breast diseases among female patients at a sub Saharan Africa tertiary hospital: a cross sectional study', Biomed central Surgery; 13(1), pp: 9.

Pukancsik, D., Kelemen, P., Ujhelyi,M., Kovacs,T., Kasler, M., Matrai,Z. (2017) 'Objective decision making between conventional and oncoplastic breast conserving surgery or mastectomy: An aesthetic and functional prospective cohort study', European Journal of Surgical Oncology; 43(2), pp: 303-310.

Reinfuss, M.,Mituś, J., Duda, K., Stelmach, A., Ryś, J., Smolak, K. (1996) 'The treatment and prognosis of patients with phyllodestumor of the breast: an analysis of 170 cases', Cancer;77(5), pp: 910-916.

Saleem, S., Javed, F., Rehman, A., Hayat, N., Bano, N., Ahmad, I\&Ahmad T. (2019) 'Oncological and Cosmetic outcome of Oncoplastic breast surgery', The Professional Medical Journal; 26(12), pp: 2222-2230.

Simmons, R.M., Cance, W.G., Lacicca, M.V. (2000) A giant juvenile fibroadenoma in a 12-year-old girl: a case for breast conservation. The Breast Journal; 6(6), pp:418-420.

Wendy, K. Ng., Mrad, M.A\& Brown, M.H. (2011) 'Juvenile fibroadenoma of the breast: Treatment and literature review', Canadian Journal of Plastic Surgery; 19 (3), pp:105-107

Yazar, S.K., Altinel, D., Serin,M., Aksoy, S and Yazar, M.(2018) 'Oncoplastic breast conserving surgery: Aesthetic satisfaction and oncologicl outcomes', European Journal of Breast Health; 14 (1), pp: 3538.

Zhang, W., Jin, Z \&Baikpour, M. (2019) 'Clinical application of ultrasound-guided percutaneous microwave ablation for benign breast lesions: a prospective study', BMC Cancer; 19(1) pp:345 